\title{
Behaviour of Glass in Cement-based Materials: Its Role on ASR
}

\author{
D. Serpa ${ }^{1, a}$, A. Santos Silva ${ }^{2, b}$, D. Soares ${ }^{2, c}$, M. Barreto Santos ${ }^{3, d}$, J. de Brito ${ }^{1, e}$
}

'DECivil-IST, Technical University of Lisbon, Av. Rovisco Pais, 1049-001 Lisbon, Portugal

${ }^{2}$ National Laboratory of Civil Engineering, Av. do Brasil 101, 1700-066 Lisbon, Portugal

${ }^{3}$ Polytechnic Institute of Leiria, Campus 2, Morro do Lena, Alto Vieiro, 2411-901 Leiria, Portugal

adiogo_serpa@hotmail.com, bssilva@Inec.pt (corresponding author), 'dsoares@Inec.pt,

‘mbarreto@estg.ipleiria.pt, ejb@civil.ist.utl.pt

\section{Abstract}

One of the most worrying degradation mechanisms in hardened concrete is the occurrence of chemical expansive reactions, namely due to alkali-silica reactions (ASR). This pathology involves the formation of expansive products inside the material. This situation is very difficult to deal with, because presently there is no efficient method to repair concrete structures affected by ASR. Hence, there is an urgent need to find preventive methods that may inhibit these reactions in new concrete structures. Nowadays, the use of pozzolanic mineral additions is recommended to mitigate this type of degradation. Moreover, their effect depends on their chemical and mineralogical composition and also on the cement content replacement ratio. Glass is a common material with some environmental problems regarding recycling and landfilling. Some studies exist about its use as fine powder, like a pozzolan, in cement-based materials. However, there are also some problems related to its use as coarse aggregate in cement-based materials due to ASR development.

This paper presents the results obtained in mortars exposed to $1 \mathrm{M} \mathrm{NaOH}$ solution prepared with crushed glass as aggregate and as addition in cement substitution. The results reveal that $A S R$ is reduced with the incorporation of glass as aggregate or addition, and its efficiency depends on its replacement content. This study shows that an incorporation of glass aggregates up to $20 \%$ in mass will generate no significant expansion when compared with concrete made with natural aggregates.

Keywords: Waste-glass, Alkali-silica reaction, Pozzolanic reaction, concrete. 